Regular Article

\title{
Thermal cycling induced room temperature aging effect in Ni-rich NiTi shape memory alloy
}

\author{
Xiebin Wang ${ }^{\text {a,* }}$, Jan Van Humbeeck ${ }^{\text {a }}$, Bert Verlinden ${ }^{\text {a }}$, Sergey Kustov ${ }^{\text {b,c }}$ \\ a Department of Materials Engineering, KU Leuven, Kasteelpark Arenberg 44 bus 2450, Leuven B3001, Belgium \\ ${ }^{\mathrm{b}}$ Departament de Física, Universitat de les Illes Balears, Cra Valldemossa km 7.5, Palma de Mallorca E07122, Spain \\ c ITMO University, Kronverkskiy av. 49, St. Petersburg 197101, Russia
}

\section{A R T I C L E I N F O}

\section{Article history:}

Received 14 October 2015

Accepted 7 November 2015

Available online $\mathrm{xxxx}$

\section{Keywords:}

Shape memory alloy

NiTi

Aging

Thermal cycling

Functional fatigue

\begin{abstract}
A B S T R A C T
This work shows that the characteristics of B19' martensite transformation in Ni-rich NiTi alloys are rather stable during aging at room temperature after quenching from $1273 \mathrm{~K}$. However, if transformation cycles between austenite and martensite are performed, the B19' martensite transformation can be strongly suppressed following aging at room temperature. As the NiTi elements normally experience cyclic transformation in applications, this phenomenon has to be taken into account as a factor for the functional degradation of NiTi alloys. Possible mechanisms of the uncovered aging effect are discussed.
\end{abstract}

(c) 2015 Elsevier Ltd. All rights reserved.
NiTi shape memory alloys are attracting increasing interest for practical applications, based on their excellent functional properties $[1,2]$. NiTi elements normally undergo cyclic phase transformation between B2 structured austenite (A) and B19' structured martensite (M) during utilization. Therefore, the stability of phase transformation behavior during cycling is of crucial practical importance. Defects, mainly dislocations, which suppress the B19' martensite transformation (MT), will be introduced during cyclic thermally induced $A \leftrightarrow M$ transformation [3-5]. It has been frequently observed that MT shifts to lower temperatures with a decreasing rate during thermal cycling $[6$, 7]. After a certain number of thermal cycles, the transformation behavior can be stabilized, due to the saturation of the defects generated by thermal cycling [5].

Aging, which can significantly affect the transformation behavior [8-11], is not expected in NiTi alloys when working at low temperatures (e.g. $<373 \mathrm{~K}$ ). In the work by Kim and Miyazaki [12], a Ti-50.9 at.\% Ni alloy was aged at $373 \mathrm{~K}$ for 125 days, and no microstructural change was found by means of transmission electron microscopy. However, it has recently been reported [13] that aging at room temperature (RT) could significantly suppress the MT, probably due to the formation of very small nano-precipitates or their nuclei during aging at RT. In Ref. [13], consecutive thermal cycling and RT-aging steps were performed on the same sample, i.e. $\mathrm{A} \leftrightarrow \mathrm{M}$ transformation cycles were performed before each RT-aging step. In this way, the possible influence of defects, which was introduced by thermal cycling, on the RT-aging effect could

\footnotetext{
* Corresponding author.

E-mail address: wangxiebin@hotmail.com (X. Wang).
}

not be detected and analyzed. In this study, a different scenario is suggested, comparing the RT-aging effect before and after thermally induced $\mathrm{A} \leftrightarrow \mathrm{M}$ transformation cycles.

A Ti-50.6 at.\% Ni alloy (nominal composition) was annealed at $1273 \mathrm{~K}$ for $2 \mathrm{~h}$, followed by water quenching. The quenched samples in the form of small disks were subjected to 3 different procedures: (A) aging of 8 samples at RT respectively for $1,2,4, \ldots 90$ days, and afterwards, the transformation behavior was characterized by differential scanning calorimetry (DSC); (B) thermal cycling of 1 sample immediately after quenching; and (C) 1 DSC cycle of 1 sample immediately after quenching followed by aging of the same sample at RT in several consecutive steps up to totally 120 days, interrupted by DSC cycles performed at $1,2,4, \ldots 120$ days after quenching. The last protocol coincides with the one used in the previous study of RT-aging [13]. The DSC measurements were performed in a TA Q2000 calorimeter with a cooling/heating rate of $10 \mathrm{~K} \mathrm{~min}^{-1}$. The microstructure of asquenched sample was analyzed by means of electron backscattered diffraction (EBSD), which revealed a bimodal grain size distribution (Fig. S1, supplementary material) with an average grain size of $41 \mu \mathrm{m}$.

All the samples subjected only to RT-aging (protocol A) show a single-stage $A \leftrightarrow M$ transformation, as summarized in Fig. 1a, where the DSC data are shown for 8 different virgin samples. The transformation peak shows an "explosive" transformation behavior, i.e. the nucleation and growth of martensite are very fast, as indicated by the vertical DSC lines observed at the onset of $A \rightarrow M$ transformation (Fig. 1a). The reason for the found "explosive" B19' MT is not clear yet, and further investigations are required to explain this phenomenon. The "explosive" transformation causes only a small scatter $(<5 \mathrm{~K})$ of MT start 

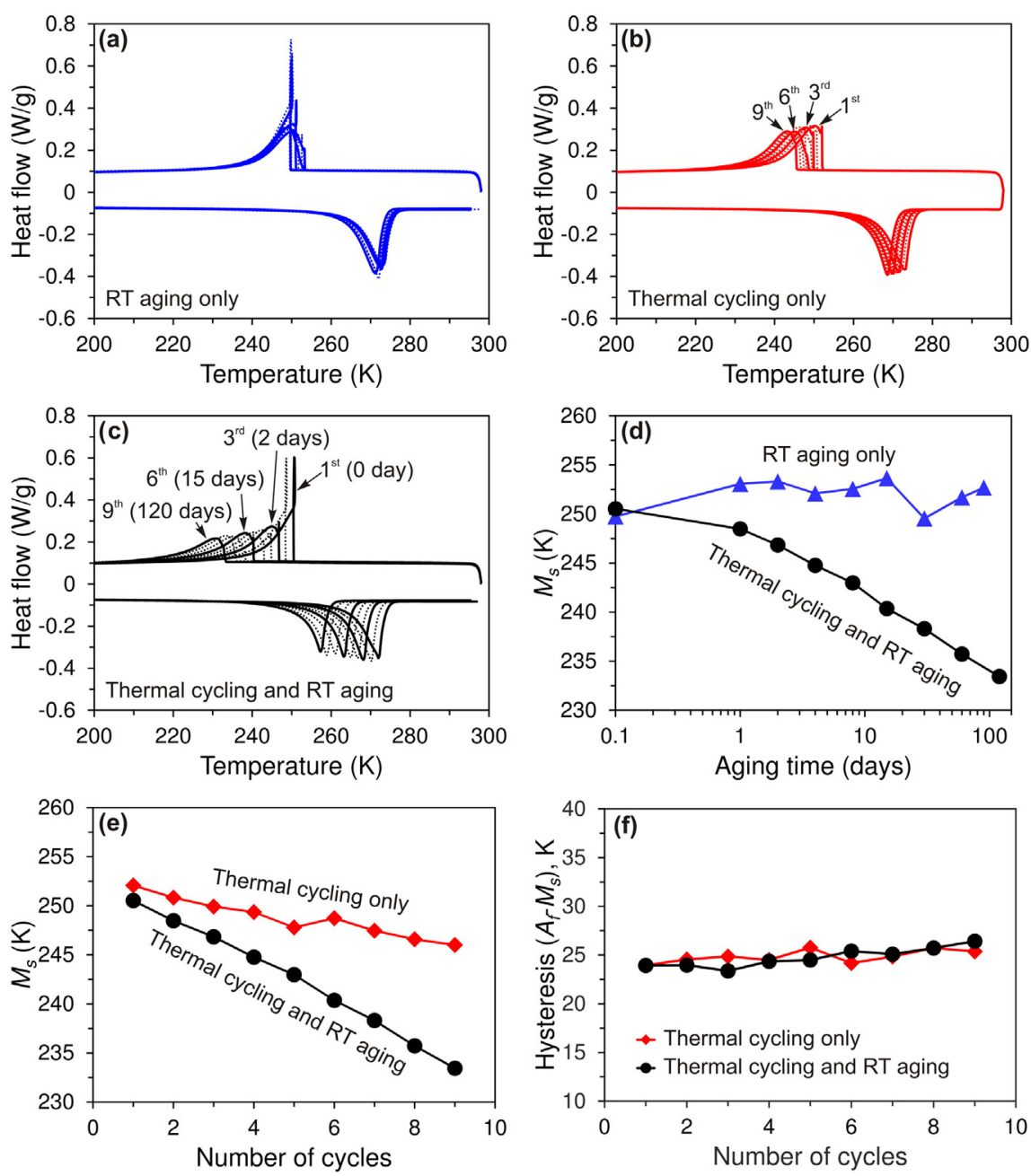

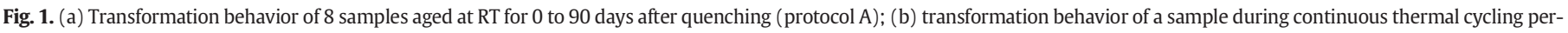

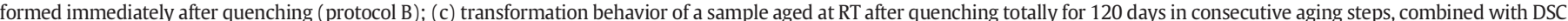

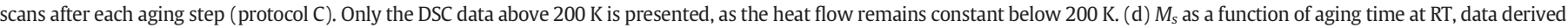

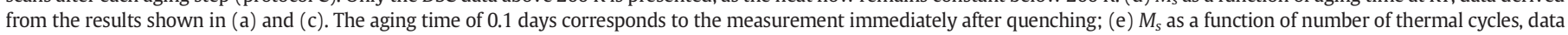

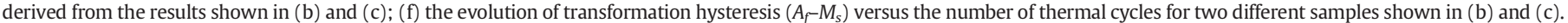

temperature $\left(M_{s}\right)$ as shown in the supplementary material (Fig. S2). $M_{s}$ of the samples aged for different durations varies between 250 and $254 \mathrm{~K}$, as shown in Fig. $1 \mathrm{~d}$. $M_{S}$ does not seem to be affected by aging.

Fig. 1b shows the transformation behavior of one sample subjected to 9 consecutive transformation cycles immediately after quenching (protocol B). The MT gradually shifts to lower temperatures with thermal cycling (from 252 to $246 \mathrm{~K}$, Fig. 1e), due to the formation of defects (e.g. dislocations) as previously reported [3-5].

Fig. 1c shows the transformation behavior of a sample that experienced the combined treatment of thermal cycling and RT-aging (protocol C). The MT shifts to lower temperatures much faster than for the samples subjected only to RT-aging (Fig. 1a) or thermal cycling (Fig. 1b). $M_{s}$ versus aging time for protocols $\mathrm{A}$ and $\mathrm{C}$ is compared in Fig. $1 \mathrm{~d}$, and $M_{s}$ versus number of thermal cycles for protocols $B$ and $C$ is compared in Fig. 1e. For combined aging-cycling treatment (protocol C), $M_{s}$ decreases from $250 \mathrm{~K}$, which was measured directly after quenching (the first thermal cycle), to $233 \mathrm{~K}$, measured after 120 days of RT-aging, interrupted by 9 thermal cycles. In contrast, the effect of only RT-aging on $M_{s}$ is not detected (Fig. 1d), and the effect of only consecutive thermal cycling remains much less intense (Fig. 1e). Fig. 1f shows that the transformation hysteresis, which is defined as the difference between $M_{s}$ and the reverse transformation finish temperature $\left(A_{f}\right)$, varies between 24 and $26 \mathrm{~K}$, indicating that the transformation hysteresis is not affected by the combined thermal cycling and RT-aging.
Fig. 2a shows the transformation behavior of a sample subjected to 12 consecutive transformation cycles directly after quenching. $M_{\mathrm{s}}$ gradually decreases from 249 to $243 \mathrm{~K}$ with thermal cycling, as indicated in Fig. 2b. Afterwards, the sample was subjected to RT-aging for 127 days, followed by one more DSC scan (the 13th cycle) shown by the red curve in Fig. 2a. The transformation behavior points to a large suppression of the MT, caused by RT-aging. $M_{s}$ drops around $7 \mathrm{~K}$ after RT-aging (for 127 days), as shown in Fig. $2 b$.

The above results indicate that only aging at RT has no detectable influence on the MT. However, the RT-aging effect could be activated by the thermal cycling, i.e. RT-aging after cyclic A $\leftrightarrow$ M transformation can suppress MT. The suppression of MT can be accelerated by increasing the aging temperature to $373 \mathrm{~K}$, as shown in the supplementary material (Fig. S3). The appearance of the R-phase (Fig. S3e) and the increase in transformation hysteresis (Fig. S3d) indicate that precipitates may form after long-term aging at $373 \mathrm{~K}[14,15]$. The temperature dependent process indicates that the uncovered RT-aging effect on the MT, mediated by thermal cycling, appears to be fundamentally similar to the MT suppression by fine $\mathrm{Ni}_{4} \mathrm{Ti}_{3}$ nanoprecipitates after "static" aging at substantially higher temperatures [9], when bulk diffusion sets in.

In the present study, a high concentration of point defects (e.g., vacancies, supersaturated $\mathrm{Ni}$ atoms) could be introduced by quenching from high temperatures $[13,16,17]$. The quenched-in defects are not stable, but a tendency to rearrange or to form precipitates is 

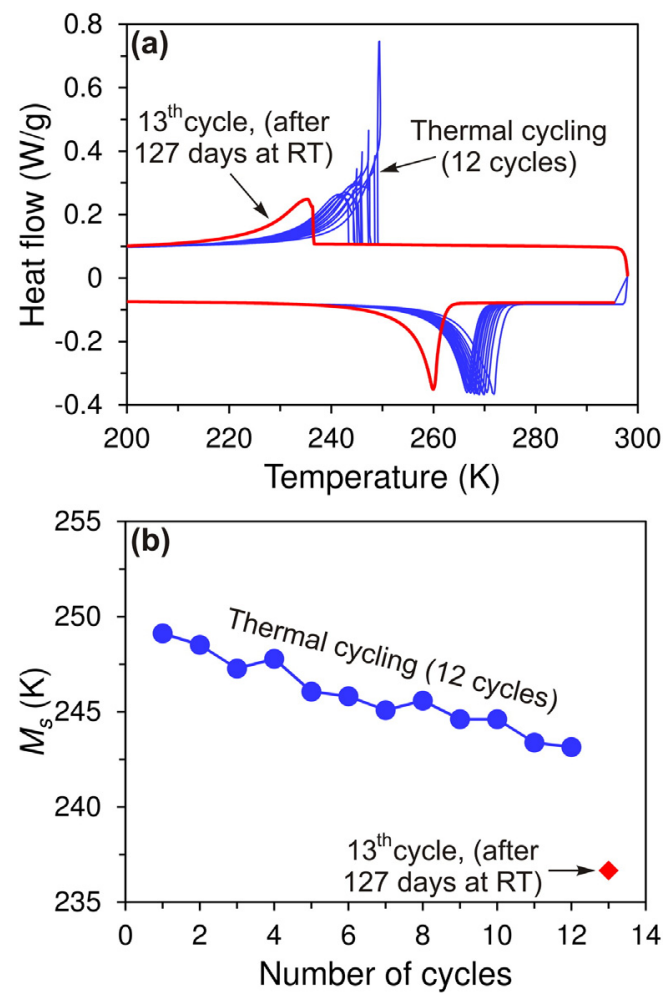

Fig. 2. (a) Transformation behavior of a Ti-50.6 at.\% Ni sample in 12 consecutive thermal cycles immediately after quenching (the blue curves) and in the 13th cycle performed after 127 days of RT-aging (the red curve). (b) Evolution of $M_{s}$ with thermal cycling, corresponding to the data in (a). (For interpretation of the references to color in this figure legend, the reader is referred to the web version of this article.)

blocked by a negligible bulk diffusion at RT. Grain boundaries may provide paths for the motion of point defects, but this effect is limited because of the low volume fraction of grain boundaries in rather coarsegrained samples with an average grain size of $41 \mu \mathrm{m}$ (Fig. S1 in the supplementary material). As a result, the MT is hardly affected only by aging at RT, as is confirmed by data in Fig. 1a and d. Therefore, the interpretation of the combined effect of aging and thermal cycling must imply activation of diffusion after thermal cycling, even at room temperature, resulting in heterogeneity of the matrix which impedes the MT.

Dislocations can be introduced by cyclic $A \leftrightarrow M$ transformation [3,4]. As a result, a microstructure enriched with dislocations and metastable point defects is formed in quenched samples after thermal cycling. The presence of dislocations could promote the motion of the point defects during the following aging at RT, probably due to the process proposed by Cottrell and Bilby [18], resulting in the segregation of quenched-in point defects on dislocation lines. The heterogeneity thus created may have higher ability to suppress the nucleation of B19' martensite, as compared with dislocations and randomly distributed point defects. However, dislocations with segregated point defects hardly affect the transformation hysteresis (Fig. 1f), indicating that this microstructure has less effect on either the motion of transformation interface or the elastic energy dissipation, which both can increase the transformation hysteresis [19]. This also suggests that no nanoprecipitates or their nuclei are formed during RT-aging for up to 120 days after thermal cycling, because an increase of transformation hysteresis is expected concurrent with a decrease of $M_{S}$ in case of formation of nanoprecipitates. Further investigations are required to understand this microstructure and related transformation behavior.

The mechanism discussed above implies a static segregation process during RT-aging, which requires bulk diffusion towards immobile dislocations, or their static pinning [20]. On the other hand, the segregation may also stem from the sweeping-up of quenched-in defects by moving interphase boundaries. This mechanism, involved in the interpretation of kinetic stabilization of martensite [21], requires diffusion of quenched-in defects together with moving interfaces, which is much more efficient than the bulk diffusion.

Although both possible mechanisms of creation the heterogeneity in quenched samples imply thermal activation, their intensity should have different dependence on the rate of MT, which triggers the RT-aging process. The static segregation process can be enhanced by the increase in the density of dislocations, which can be caused by a higher $\mathrm{A} \leftrightarrow \mathrm{M}$ transformation rate. In contrast, the higher is the transformation rate, the less efficient is sweeping-up of quenched-in defects by moving interfaces and the less efficient is the aging in suppression of the MT. In future experiments, this difference in the effect of the MT rate could serve to clarify the specific mechanism involved.

In summary, this work shows that the transformation characteristics of NiTi alloys are rather stable at RT, even after quenching from rather high temperatures, during "static" RT-aging without combining with transformation cycles. However, a remarkable suppression of the MT is observed during RT-aging combined with thermal cycling. Two feasible mechanisms are suggested, i.e. "static" segregation of quenched-in defects on dislocations and sweeping-up of quenched-in defects by moving interphase boundaries. However, further investigations are required to fully understand the phenomenon uncovered. The results discussed in this work suggest that the stabilization of MT temperatures by thermal cycling has to be reconsidered, especially for the samples quenched from slightly higher than usual temperatures (e.g. $1273 \mathrm{~K}$ ).

This work was supported by the Research Foundation Flanders (FWO) under Grant No. G036615N. X. Wang wishes to thank the China Scholarship Council (Grant No. 2010622037) for financial support. S. Kustov acknowledges the support from Spanish MINECO and FEDER (Project MAT2014-56116-C4-1-R) and from Russian Federation (Grant 074-U01) through ITMO Visiting Professorship program. The assistance from Mr. Liugang Chen with the heat treatments is highly appreciated.

\section{Appendix A. Supplementary data}

Supplementary data to this article can be found online at http://dx. doi.org/10.1016/j.scriptamat.2015.11.007.

\section{References}

[1] J. Van Humbeeck, Mater. Sci. Eng. A 273-275 (1999) 134-148.

[2] K. Otsuka, T. Kakeshita, MRS Bull. 27 (2002) 91-98.

[3] J. Zhang, C. Somsen, T. Simon, X. Ding, S. Hou, S. Ren, X. Ren, G. Eggeler, K. Otsuka, J. Sun, Acta Mater. 60 (2012) 1999-2006

[4] T. Simon, A. Kroger, C. Somsen, A. Dlouhy, G. Eggeler, Acta Mater. 58 (2010) 1850-1860.

[5] S. Miyazaki, Y. Igo, K. Otsuka, Acta Metall. 34 (1986) 2045-2051.

[6] X. Wang, B. Verlinden, J. Van Humbeeck, Intermetallics 62 (2015) 43-49.

[7] M.F.-X. Wagner, S.R. Dey, H. Gugel, J. Frenzel, C. Somsen, G. Eggeler, Intermetallics 18 (2010) 1172-1179.

[8] K. Otsuka, X. Ren, Prog. Mater. Sci. 50 (2005) 511-678.

[9] X. Wang, S. Kustov, K. Li, D. Schryvers, B. Verlinden, J. Van Humbeeck, Acta Mater. 82 (2015) 224-233.

[10] Y. Zheng, F. Jiang, L. Li, H. Yang, Y. Liu, Acta Mater. 56 (2008) 736-745.

[11] M.C. Carroll, C. Somsen, G. Eggeler, Scr. Mater. 50 (2004) 187-192.

[12] J.I. Kim, S. Miyazaki, Acta Mater. 53 (2005) 4545-4554.

[13] S. Kustov, B. Mas, D. Salas, E. Cesari, S. Raufov, V. Nikolaev, J. Van Humbeeck, Scr. Mater. 103 (2015) 10-13.

[14] W. Tirry, D. Schryvers, Nat. Mater. 8 (2009) 752-757.

[15] H. Shi, S. Pourbabak, J. Van Humbeeck, D. Schryvers, Scr. Mater. 67 (2012) 939-942.

[16] Y. Nagai, T. Toyama, Z. Tang, M. Hasegawa, H. Matsumoto, S. Hanada, Scr. Mater. 54 (2006) 1751-1753.

[17] S. Kustov, S. Golyandin, K. Sapozhnikov, M. Morin, Scr. Mater. 43 (2000) 905-911.

[18] A.H. Cottrell, B.A. Bilby, Proc. Phys. Soc. A 62 (1949) 49-62.

[19] R.F. Hamilton, H. Sehitoglu, Y. Chumlyakov, H.J. Maier, Acta Mater. 52 (2004) 3383-3402.

[20] S. Kustov, J. Pons, E. Cesari, J. Van Humbeeck, Acta Mater. 52 (2004) 3075-3081.

[21] S. Kustov, J. Pons, E. Cesari, J. Van Humbeeck, Acta Mater. 52 (2004) 3083-3096. 